\title{
Roles of non-coding RNAs in stress response in plants
}

\author{
M. Chen ${ }^{1 *}$, J. Wang ${ }^{1}$, O.B. Dobrovolskaya ${ }^{2,3}$, V.N. Babenko ${ }^{2,3}$, Y.L. Orlov², \\ Y. Liu ${ }^{1}$, L.S. Samarina** \\ ${ }^{1}$ Zhejiang University, Hangzhou, China \\ ${ }^{2}$ Novosibirsk State University, Novosibirsk, Russia \\ ${ }^{3}$ Institute of Cytology and Genetics SB RAS, Novosibirsk, Russia \\ ${ }^{4}$ FSBSI Russian Research Institute of Floriculture and Subtropical Crops, Sochi, Russia \\ *e-mail:mchen@zju.edu.cn,q11111w2006@ya.ru
}

Key words: non-coding RNA, transcriptomics, stress response, crop plants

Motivation and Aim: Eukaryotic genomes encode thousands of non-coding RNAs (ncRNAs), which play crucial roles in transcriptional and post-transcriptional regulation of gene expression. The computer analysis of transcription regulation in stress response in crop plants is a challenging problem. Accumulating evidence indicates that ncRNAs, especially microRNAs (miRNAs) and long ncRNAs (lncRNAs), have emerged as key regulatory molecules in plant stress responses. We have summarized the current progress on the understanding of plant miRNA and lncRNA identification, characteristics, bioinformatics tools, and resources, and provided examples of mechanisms of miRNAand lncRNA-mediated plant stress tolerance [1]. Although remarkable progress has been made in explaining the role of plant miRNAs and lncRNAs in plant adaption to stress, mechanistic details are still limited.

Methods and Algorithms: With the advantage of the next-generation sequencing technologies and bioinformatics approaches, a great number of ncRNAs have been identified and characterized in plants, especially miRNAs and lncRNAs. miRNAs and lncRNAs are two important types of ncRNAs in plants, which play important roles in various biological processes. Rapid progress in high-throughput sequencing and advancement of bioinformatics tools provide revolutionary ways for identification and prediction of novel ncRNAs.

Results: In this work, we summarized the common bioinformatics tools and resource of miRNAs and lncRNAs. In addition, recently-developed single-cell sequencing and single-molecule sequencing will offer more opportunities to increase the number of ncRNAs. Therefore, it is necessary to develop new bioinformatics methods for the identification and functional analysis of ncRNAs.

Conclusion: Recent works show role of alternative splicing events in stress (draught) response in bread wheat [2]. We continue work on integration of stress-response data and gene sets using available data sources.

Acknowledgements: The research has been supported by RFBR 18-04-00483. Computing done at Siberian Supercomputer center SB RAS was supported by budget project 03242018-0017.

\section{References}

1. Wang J., Meng X., Dobrovolskaya O.B., Orlov Y.L., Chen M. (2017) Non-coding RNAs and their roles in stress response in plants. Genomics Proteomics Bioinformatics. 15(5):301-312.

2. Liu Z. et al. (2018) Global profiling of alternative splicing landscape responsive to drought, heat and their combination in wheat (Triticum aestivum L.). Plant Biotechnol J. 16(3):714-726. 